This document is the accepted manuscript version of the following article: Boesel, $L$. F., de Geus, M., \& Thöny-Meyer, L. (2013). Effect of PLA crystallization on the structure of biomimetic composites of PLA and clay. Journal of Applied Polymer Science, 129(3), 1109-1116. https://doi.org/10.1002/app.38698

\title{
Effect of PLA crystallization on the structure of biomimetic composites of PLA and clay
}

\author{
Luciano F. Boesel, Matthijs de Geus*, Linda Thöny-Meyer
}

EMPA - Swiss Federal Laboratories for Materials Science and Technology, Laboratory for Biomaterials, Lerchenfeldstrasse 5, 9014, St. Gallen, Switzerland

*Corresponding author: Matthijs.DeGeus@empa.ch, Tel: +41587657674

Dated: 28.01 .2021

ABSTRACT: Composites of poly(lactic acid) (PLA) and organoclays with clay loadings of up to $80 \%$ were prepared as self-supporting films using a doctor-blading approach. Depending on the properties of the used organoclay, either intercalated nanocomposites or conventional composites were obtained. The incorporation of such high amounts of clay resulted in up to tenfold decrease in the water vapor transmission rate when compared to the pristine polymer. The effect of clay platelets on the crystallization of PLA chains was also studied; it was found that high amounts of clay hinder only the melt crystallization of the polymer, while cold crystallization proceeds as usual. On the other hand, the crystallization of PLA also influenced the composite structure by increasing the extent of intercalation of polymer between clay layers. This study thus shows that the change in the extent of clay-polymer interactions is also an important factor in controlling nanocomposite structure, especially for high loading.

Keywords: layered structures, nanoclays, transport properties, biopolymers, nanocomposites

\section{Introduction}

The preparation of composites of polymers with layered particles has been in recent years a common strategy to improve mechanical, thermal, and barrier properties of polymers. Usually, small amounts (ca. $5 \mathrm{wt} \%$ ) of layered materials such as clays or layered double hydroxides are mixed with a polymer matrix during melt processing in a twin-screw extruder, leading to the exfoliation and random dispersion of platelets in the matrix. Such strategy has been applied to improve the barrier properties of poly(lactic acid) (PLA), a widely used biopolymer which, however, suffers from inadequate properties in comparison to standard packaging materials such as polyethylene (PE) and polyethyleneterephthalate (PET). Compared with the latter, the

permeability of PLA is about 11 times (for water vapor) or 9 times (for oxygen) larger. ${ }^{1}$ The 
incorporation of clay particles in the PLA matrix during melt processing resulted in the improvement of barrier properties of PLA by about $60 \%{ }^{2-4}$ However, melt processing presents some drawbacks, specially if improvements in the barrier properties are concerned. It is well known that the state of dispersion and the orientation of clay minerals in the polymer matrix play a key role in the achievable barrier properties of materials. Due to aggregation of individual clay nanoplatelets, barrier properties initially improve, but, at a certain clay volume fraction (which can be as low as $1,4 \%$ ), permeability may increase due to the lack of exfoliation or the decrease of effective aspect ratio of clay. ${ }^{5}$ Moreover, randomly oriented platelets provide only half the improvement in barrier properties in comparison to platelets perfectly aligned with the surface of the film. ${ }^{6}$ Therefore, melt compounding of polymers with clay is expected to show limitations in the ability to improve barrier properties, because aspect ratio may be reduced, clay platelets may agglomerate, and platelet orientation is usually random.

An alternative method of improving the barrier properties is by applying a coating over the base film, for example a silicon oxide coating, ${ }^{7}$ or layer-by-layer assembly of organic and inorganic materials ${ }^{8 ; 9}$. A similar approach is to form a composite coating with the same polymer matrix and layered particles as the dispersed phase. Due to the similarity of the structures so obtained with those of nacre (a natural, layered composite found in mollusk shells), these layerby-layer assemblies are also known as "biomimetic composites". Although the optimization of the interface between polymer and particle and of the inorganic content of such composites has led to dramatic improvements of mechanical and barrier properties of a diversity of materials, the requirement for tenths of alternate dip- or spin- coating processes, washing and drying steps, makes this methodology less interesting for large-scale industrial production.

Recently, an alternative technique has been developed that allows high throughput fabrication of such biomimetic composites within a one-step process. ${ }^{10}$ In this method, a polymer solution and a clay dispersion are mixed allowing the polymer to adsorb on the clay surface. The mixture is then applied in a single step through doctor-blading to allow the preparation of self-supporting films or coatings onto another polymer substrate. Although the ordering and alignment of clay platelets in this case is worse than that obtained in a layer-by-layer process, ${ }^{10}$ the easiness of application make this process well suitable for industrial purposes.

The presence of high amounts of clay platelets in such biomimetic composites is expected to change the crystallization extent and kinetics of the matrix polymer. In fact, it is well accepted that clay platelets act as nucleating agent for the crystallization of polymers $4 ; 11$. This has already 
been demonstrated for PLA ${ }^{12-14}$. However in these previous studies conventional nanocomposites containing low amounts of clay (less than 10\%) were used, which did not adequately simulate the environment in a biomimetic composite, that usually contains above $70 \mathrm{wt} \%$ clay minerals $9 ; 10$. On the other hand, annealing of a polymer in the interlayer of clay platelets may lead to changes in the microstructure of the composite. During annealing, the degree of crystallinity increases, and the crystal form may also change, depending on the temperature at which annealing is performed. Specifically regarding PLA, it has been demonstrated that annealing at low temperatures (about $120^{\circ} \mathrm{C}$ ) leads to a mixed population of $\alpha$ and $\alpha^{\prime}$ crystals, which is different from those formed after solvent casting. ${ }^{15}$

In this paper, we applied the one-step process for the preparation of highly loaded PLA/clay composites. Due to the absence of strong ionic interactions between both components, the preparation of an ordered, layered material in this system was a greater challenge than when dealing with hydrophilic polymers. We subsequently performed an extensive characterization of the interactions between clay and polymer and on the crystallization phenomena of the polymer when in contact with the clay, in order to elucidate how the presence of clay influences the crystallization of PLA and how the crystallization influences the clay-polymer interactions. For very high amounts of clay (about $80 \%$ ), an interesting phenomena was observed: PLA was not able to melt-crystallize, although cold-crystallization did occur.

\section{Experimental}

\subsection{Materials}

The PLA used in this work was acquired from NatureWorks, USA. It is a PLLA containing about 1,4 \% D-lactic acid units. Two types of organomodified layered silicates (OMLS), also called organoclays, were used: Dellite 43B (montmorillonite modified with dimethylbenzyltallow ammonium ion, supplied by Laviosa Chimica Mineraria S.p.A., Italy), and Nanomer I.34TCN (montmorillonite with methyldihydroxyethyltallow ammonium ion, supplied by Sigma-Aldrich). They are referred as "43B" and "I34", respectively, in the rest of this paper. Other solvents were of reagent-grade and used as received. 


\subsection{Preparation and application of composites}

PLA was initially vacuum-dried at $80{ }^{\circ} \mathrm{C}$ during $4 \mathrm{~h}$. Melt compressed PLA plates (approximately $0,5 \mathrm{~mm}$ thick) were prepared at $210{ }^{\circ} \mathrm{C}$ to serve as support for the coatings. A dispersion of OMLS in chloroform $(0,5 \% \mathrm{w} / \mathrm{v})$ was slowly added to a solution of $5 \% \mathrm{w} / \mathrm{v}$ of PLA in chloroform, and the mixture was stirred overnight. The relative amounts of solution and dispersion were varied in order to obtain composites containing $20 \%$ or $50 \%$ of PLA (named PLA20 and PLA50, respectively), as well as pure PLA films (named PLA100). The solvent was subsequently evaporated until a final solids concentration of approximately $15 \%$ was reached. The mixture was then poured over the substrate (poly(tetrafluoroethylene) sheets for selfsupporting films, or PLA plates for coatings) and spread by using a bar-coater at a drawing speed of $15 \mathrm{~mm} / \mathrm{s}$. The excess solvent was initially dried at room temperature; residual solvent was then removed by vacuum-drying for $24 \mathrm{~h}$ at room temperature.

\subsection{Characterization}

Fourier-transformed infrared spectroscopy (FTIR) was performed with the Attenuated Total Reflectance (ATR) module. Both specimens and background spectra were collected at a $4 \mathrm{~cm}^{-1}$ resolution by adding 64 spectra. For the second part of this study (see "Results and Discussion"), 3 or 4 specimens from different batches were analyzed, and for each absorbance band analyzed the median from all values was considered.

Differential scanning calorimetry (DSC) was performed using a Mettler-Toledo DSC822 $2^{e}$ apparatus. The following 3-step program was applied to all specimens: first heating from $20{ }^{\circ} \mathrm{C}$ to $200{ }^{\circ} \mathrm{C}$ at $10{ }^{\circ} \mathrm{C} / \mathrm{min}$; cooling to $20{ }^{\circ} \mathrm{C}$ at a cooling rate of $-2{ }^{\circ} \mathrm{C} / \mathrm{min}$; second heating to $200{ }^{\circ} \mathrm{C}$ at $10{ }^{\circ} \mathrm{C} / \mathrm{min}$. The glass transition temperature $\left(T_{g}\right)$ and the melting temperature $\left(T_{m}\right)$ were obtained from the first heating cycle; the degree of crystallinity $\left(\chi_{c}\right)$ was calculated from the enthalpy of fusion of the second heating cycle (as prepared films) or the first heating cycle (annealed films) according to:

$$
\chi_{C}=100 * \frac{\Delta H_{m}}{C_{P L A} * \Delta H_{m}^{0}}
$$


where $C_{P L A}$ is the mass fraction of PLA in the formulation, $\Delta H_{m}$ is the enthalpy of fusion of the specimen, and $\Delta H_{m}^{0}$ is the enthalpy of fusion of a $100 \%$ crystalline PLA $(94 \mathrm{~J} / \mathrm{g}){ }^{16}$.

Scanning electron microscopy (SEM) was performed in a Hitachi S-4800 instrument, using an acceleration voltage of between $2 \mathrm{kV}$ and $10 \mathrm{kV}$. Before observation, specimens were coated with a thin $(\sim 5 \mathrm{~nm})$ gold layer in a Polaron E5100 sputtering machine.

Water vapor transmission rate was measured according to ASTM Standard E-96 using the "water method". The area of the films was adjusted by using aluminum masks with the desired area. Tests were carried out at an external relative humidity of $50 \%$ and temperature of $23{ }^{\circ} \mathrm{C}$.

X-ray diffraction (XRD) was performed in a Philips PW 1729/1820 apparatus, using $\mathrm{Cu} \mathrm{K \alpha}$ radiation $(1,54 \mathrm{~nm})$. The conditions used for all specimens were: scanning between $1,9^{\circ}$ and $40^{\circ}$ $(2 \Theta)$, with a step size of $0,03^{\circ}(2 \Theta)$ and $10 \mathrm{~s} / \mathrm{step}$. Powder samples were analyzed with the texture technique in order to improve the intensity of $00 l$ reflections of clay layers. Films were directly glued on the substrate with vaseline. Talc (with three main reflections at $9,5^{\circ}, 19^{\circ}$, and $28,7^{\circ}$ ) was used as an internal standard to adjust the position of the reflections of all specimens.

Thermogravimetric analysis (TGA) was performed in a Netzsch TG209F1 apparatus. Dynamic experiments were conducted from $25{ }^{\circ} \mathrm{C}$ to $800{ }^{\circ} \mathrm{C}$ with a heating rate of $20{ }^{\circ} \mathrm{C} / \mathrm{min}$.

\section{Results}

\subsection{Preparation and characterization of biomimetic composites of PLA and organoclay}

Figure 1 displays cross-sections of self-supporting films made of pure PLA and composites. There is a clear change in the microstructure of both materials, with PLA showing a bulk, monolithic structure typical of neat polymers. By contrast, the composite showed a layered structure denoting a strong alignment of polymer and clay layers. This alignment is caused by the high shear forces originating during the doctor-blading process with a concentrated slurry and the high-aspect ratio of clays, which tend to align parallel to the film surface. Figure 1 also shows an optical micrograph of a composite containing $80 \%$ of clay platelets; despite the high loading, the film $(30 \mu \mathrm{m}$ thickness $)$ still shows a remarkable transparency. As shown by Yao and 
coworkers, ${ }^{17}$ the transparence of biomimetic composites arises from the high orientation of the polymer-clay building blocks, which decreases the light scattering between the clay particles. In contrast, clay platelets in conventionally prepared composites are randomly dispersed, which contributes to an increased light scattering. 17

Figure 2 shows XRD spectra of some of the produced materials. We have in this work used two organoclays with different organic cations and, therefore, different interlayer space distances $\left(d_{001}\right)$ and solubility parameters $(\delta)$. Due to the presence of hydroxyl groups in the organic cation, clay I34 is more hydrophilic and posseses a higher $\delta\left(\sim 20(\mathrm{MPa})^{1 / 2}\right)$, which is closer to that of PLA $\left(\sim 21(\mathrm{MPa})^{1 / 2}\right)$ than clay 43B $\left(\sim 18(\mathrm{MPa})^{1 / 2}\right)^{18 ; 19}$. Unexpectedly, the miscibility of PLA with I34 was not better than with 43B, as shown in Figure 2. The basal spacing of clay I34 after incorporation of $50 \%$ of PLA was identical to that for the pristine clay $(1,8 \mathrm{~nm})$, that is, no intercalation was observed in these composites. However, composites containing clay 43B showed a peak corresponding to the intercalation of PLA chains between clay platelets $(3,0 \mathrm{~nm})$, together with a shoulder corresponding to the basal spacing of the pristine clay $(2,1 \mathrm{~nm})$. These results show that the interlayer spacing of the clay plays a more important role in the final structure achieved by nanocomposites prepared by solution intercalation than the miscibility of the organic modifier with the polymer. In a similar study, but with lower clay content, Ojijo and co-workers ${ }^{20}$ found similar results for melt-processed blends based on PLA: in that case, a higher interlayer spacing favored intercalation and exfoliation to a larger extent than did the miscibility of the modifier with PLA.

Thermogravimetric analysis showed that the incorporation of organoclay into PLA decreased the onset temperature of mass loss (Figure 3). This apparent decrease of thermal resistance, however, is not linked to a deleterious effect of the clay on PLA, but to the presence of the organomodifier itself, which has a low degradation temperature. ${ }^{21}$ The presence of aromatic units in the organocation has been linked to a less stable organoclay; ${ }^{21}$ accordingly, we found that clay 43B (which contains aromatic units) is less stable than clay I34. As shown in Figure 3, the organic cations started to degrade at about $200{ }^{\circ} \mathrm{C}$ (clay 43B) or $250{ }^{\circ} \mathrm{C}$ (clay I34), the same temperatures observed for the onset of degradation of the respective composites with PLA. In fact, both composites presented a two-step mass loss, the first one corresponding to the organic cation of the OMLS, and the second one corresponding to PLA itself. This can be easily observed when comparing the curve for the composite PLA/43B with that of the pure components. Despite 
this decreased thermal resistance, it should be stressed that differences in the curves were only apparent for temperatures higher than $150{ }^{\circ} \mathrm{C}$, which is much higher than typical application temperatures for PLA. Therefore, no preclusion of normal PLA functioning is to be expected due to the incorporation of clay.

On the other hand, flame resistance was greatly improved by the incorporation of clay particles. As can be seen in the Supporting Information videos, the composite with $50 \%$ of $43 \mathrm{~B}$, although burning when in contact with a blue-flame, conserved its original shape. This behavior can be explained by the initial burn-out of the polymer fraction, with a consequent formation of a dense armored "skin" composed of silicate and char from the polymer burning. A similar behavior has been observed for other polymers. 10;22 Pure PLA started to drop immediately after burning and lost structural integrity in a few seconds. Therefore, the developed layered composites could be used as a protective shield for PLA when submitted to high temperatures.

Last, we also evaluated the barrier properties of the developed biomimetic composites, focusing on water vapor barrier properties. As already mentioned in the Introduction, the barrier properties of PLA are much worse than those of PET, a conventional polyester the properties of which are typically considered as a benchmark for PLA. We then tested our composites both as free-standing films, with a high amount of clay particles (80 wt\%), or as coatings on PLA plates. For the latter, the maximum amount of particles that still allowed a homogeneous coating to be formed was $50 \mathrm{wt} \%$. Table 1 shows the best results obtained for each situation. Improvements of up to 10 fold in the original barrier properties were obtained for free-standing films. Even coatings with a much lower clay amount were still successful in reducing water permeability, although the improvements were more modest. Although the composites had a different structure, as seen by XRD, no significant differences were observed with respect to the barrier properties for formulations containing the same amount of clay. By contrast, the amount of clay played a significant role in the achievable barrier properties, with $80 \mathrm{wt} \%$ (the highest loading prepared by us) providing the largest improvement. Last, it is also interesting to note that PLA coated with a thin (thinner than $5 \mu \mathrm{m}$ ) layer of biomimetic composite presented properties similar to those of a free-standing film of the same material. This data clearly shows that significant improvements in barrier properties of PLA can be obtained with a fast and straightforward coating method. This technique avoids changes in the processing conditions of PLA and keeps the integrity of clay platelets, one of the main challenges when preparing nanocomposites via melt processing. 


\subsection{Crystallization of PLA in contact with clay}

In the second part of this work, we studied the crystallization behavior of PLA when mixed with varying amounts of clay. In contrast to other studies $12-14 ; 23-25$, we focused on highly loaded composites, i.e., those containing between $50 \%$ to $80 \%$ of clay on a weight basis. We performed this extended characterization only with composites containing clay Dellite 43B. For that, we employed DSC, FTIR and XRD analyzes, both before and after annealing all materials at $130{ }^{\circ} \mathrm{C}$ for one hour under vacuum. The annealing temperature was chosen to be above the cold crystallization temperature found by DSC (between $100{ }^{\circ} \mathrm{C}$ and $110{ }^{\circ} \mathrm{C}$ ). These crystallization conditions simulate those in a first heating run in DSC experiment, therefore all samples crystallized, and the degree of crystallinity decreased with increasing clay content, as we will discuss later.

The FTIR spectra of specimens after annealing showed mostly two differences when compared to the same materials before annealing: in the carbonyl symmetrical stretching of PLA (at about $1750-1760 \mathrm{~cm}^{-1}$ ) and the Si-O stretching of clay minerals (at about $1000 \mathrm{~cm}^{-1}$ ). A detailed view of these regions is displayed in Figures $4 \mathrm{a}$ and $4 \mathrm{~b}$. Table 2 lists average values for the position of the bands, taken from 3-4 specimens of each composition. Clearly, annealing had different effects on the compositions: while for PLA20 a greater change was observed in the Si-O stretching region, for the other films the change was more prominent in the carbonyl stretching band. After annealing, peak splitting was observed for all compositions, although in PLA20 only a small shoulder could be detected at $1750 \mathrm{~cm}^{-1}$. As we will discuss later, this peak splitting originates from the crystallization of PLA into $\alpha$ - or $\alpha$ - phases ${ }^{15}$.

XRD spectra of all compositions are shown in Figure 5, both before and after annealing. On the PLA100 spectrum, peaks corresponding to a stereocomplex between PDLA and PLLA can be identified at $21,5^{\circ}$ and $24^{\circ} 26 ; 27$. After annealing, such peaks decreased in intensity, and new ones, assigned to the $\alpha^{\prime}$ phase of PLA appeared at $16,6^{\circ}$ and $18,9^{\circ} 26$. Simultaneously, the intensity and area of the amorphous halo decreases. The same behavior was observed for both composites; moreover, for PLA20 the peak corresponding to the (001) of the organoclay (at $4,2^{\circ}$ ) disappeared after annealing, giving rise to two new ones which match those found for PLA50 
both before or after annealing (at $2,8^{\circ}$ and $5,1^{\circ}$ ). For PLA20, other peaks in the spectra corresponded to those of the organoclay.

Figure 6 shows the calorigrams of pure PLA and one selected composite. The endothermic peak near $T_{g}$ was representative for all compositions analyzed, with or without clay. It appeared only in the first heating cycle, being caused by the physical aging of PLA below $T_{g}$ and the consequent excess enthalpy of relaxation that is released when the material reaches $T_{g}{ }^{27 ; 28}$ On the other hand, only pure PLA showed a double melting peak, both in the first and in the second heating cycle for unannealed samples (inset in Figure 6), and in the second heating cycle for annealed ones. These double melting peaks are the result of simultaneous melting and recrystallization of crystals at temperatures between cold crystallization and melting. ${ }^{28} \mathrm{~A}$ summary of the important thermal properties of all compositions obtained by DSC of as-prepared and annealed samples is given in Table 3. For PLA20 specimens, the measurement of $T_{g}$ was hindered by an endothermic peak from the clay at about $40-50{ }^{\circ} \mathrm{C}$; this correlates well with the melting temperature of paraffinic substances, and the pure organoclay displays this peak at $43{ }^{\circ} \mathrm{C}$. The broad endo/exo-therm peak observed before $T_{m}$ of annealed specimens results from the overlapping of two contributions: $\alpha^{\prime}$-to- $\alpha$ transition upon heating (exothermic) and meltrecrystallization of the $\alpha$-crystal formed in the previous annealing process ${ }^{15}$. These transitions were also found in PLA100 and PLA20.

From DSC curves, we determined a very low degree of crystallinity of the as-prepared film: $\sim 2 \%$ for PLA and less than $1 \%$ for the composites. This is also clearly observed in Figure 5, where PLA100 and PLA50 display a large amorphous halo and only two small peaks corresponding to the stereocomplex. Both the halo and the peaks almost disappear in PLA20.

\section{Discussion}

The carbonyl stretching of PLA has been reported to be sensitive to the conformation, intra- and intermolecular interaction of PLA chains. ${ }^{15 ; 29 ; 30}$ Moreover, it has also been shown that this band is shifted towards lower wavenumbers due to hydrogen bonding. ${ }^{29 ; 31}$ It has been observed that amorphous PLA presents two broad bands at about $1758 \mathrm{~cm}^{-1}$ and $1776 \mathrm{~cm}^{-1}$ (very weak); PLLA homopolymer has a quadruple peak with a main contribution at about $1758 \mathrm{~cm}^{-1}$ and 
$1749 \mathrm{~cm}^{-1}$; and a stereocomplex between PLLA and PDLA has a single peak centered at about $1745 \mathrm{~cm}^{-1}$. 29;32 The position of the carbonyl band for PLA100 is in agreement with what would be expected for a stereocomplex, thus confirming the information provided by the XRD spectrum in Figure 5. By contrast, composites show a displacement of this band towards higher frequencies, despite the appearance of the stereocomplex peaks (at a very low intensity, especially for PLA20). The displacement of the carbonyl band towards lower wavenumbers is assigned to the occurrence of hydrogen bonding (present in stereocomplexes, but not in homocrystals of PLA) $29 ; 31$. It is surprising that the bands (in FTIR spectra) and peaks (in XRD spectra) assigned to the stereocomplex appear at all, given the very low amount of D-units in the raw material $(\sim 1,4 \%$, according to the manufacturer) and the low crystallinity of the as-prepared samples (calculated from the DSC curves). Tsuji ${ }^{33}$ reported that during the course of solvent evaporation, the polymer concentration exceeds first the critical level of stereocomplex crystalline formation, and only then that of homocrystalline formation. Therefore, and despite the low amount of D-units, stereocomplex crystals are predominantly formed, and we observed no homocrystals in the as prepared films.

The increased wavenumber of the carbonyl stretching (for PLA20) may be attributed to decreasing hydrogen bonding and decreasing extent of stereocomplex formation in the matrix. That would be expected if PLA chains are not able to pack close together during solvent casting due to the high amount of clay platelets present in the medium; instead, interactions between PLA chains and clay platelets would be favored to the detriment of PLA intermolecular interactions. This is confirmed by the position of $\mathrm{Si}-\mathrm{O}$ vibrations of clay platelets. These vibrations are very sensitive to hydrogen bonding with water or organic molecules. Variations in the frequency of the Si-O absorption may be due to changes in the Si-O bond length or the Si-OSi bond angle, which appear when the basal oxygen atoms of the silica tetrahedra form hydrogen bonds with molecules in the interlayer ${ }^{34}$. As seen in Table 2 and Figure $4 b$, only PLA20 showed a change in the position of this band. In PLA50, this band was approximately at the same position as for the organoclay (considering a resolution of $4 \mathrm{~cm}^{-1}$ used in all experiments). PLA20 was also the one with lower intrachain hydrogen bonding in PLA, as inferred from the carbonyl stretching vibration. It is then clear from this data that as the clay content increases, there is a tendency for increased clay-polymer rather than polymer-polymer interactions.

Despite this increased interaction, the amount of PLA in PLA20 was not sufficient to increase the interlayer spacing of the clay. As seen in Figure 5, the $d_{001}$ of the composite matched that of 
the organoclay. With PLA50, a different behavior was observed: in this case, intercalation of PLA did occur (as already shown in Section 3.1), although the original (001) reflection of the clay was still visible as a shoulder to the main peak. Interestingly, this intercalation did not lead to a stronger polymer-clay interaction, as shown by the FTIR data.

The presence of the clay leads also to different thermal behavior, as depicted in Figure 6 and Table 3. Both composites presented an almost $10{ }^{\circ} \mathrm{C}$ higher $T_{g}$ when compared to that of the neat polymer. The increased $T_{g}$ represents a well-known phenomena in which the presence of hard clay platelets hinders long-range movements of amorphous chains. ${ }^{4 ; 11}$ It has also been reported that $T_{m}$ does not change significantly with the addition of clay particles. ${ }^{4}$

The main difference among the several formulations was in the crystallization behavior. The typical double melting peak of PLA was not present in any composite, an indication that the melting-recrystallization phenomenon was hindered by the presence of clay. That is, crystals in the composite are more stable than those in neat PLA ${ }^{28}$, probably due to a protecting effect of the clay platelets. Moreover, PLA20 was not able to crystallize during cooling from the melt, while PLA50 achieved a degree of crystallinity similar to that of the neat polymer. This supports our previous assumption of an increased interaction between PLA chains and clay platelets as compared to intramolecular PLA interactions. During the first heating run in the DSC measurement, the material structure is controlled by that formed during solvent casting and the mobility of chains is very limited, therefore chains which were already in close contact would be able to crystallize. From the crystallization peak, about $8 \%$ of the PLA in PLA20 was able to crystallize, while that value increased to about $33 \%$ of the PLA in PLA100 and PLA50. However, after melting, PLA chains are more mobile and able to reorganize in order to maximize interactions with clay particles, thereby hindering the ability of PLA to crystallize. This is in strong contrast with the behaviour of PLA50 which not only crystallizes to a higher extent than cold crystallized PLA50, but achieved levels of crystallinity similar to those of PLA100.

The interactions between PLA and clay platelets, therefore, are less favorable than PLA-PLA interactions but once formed, are able to hinder the latter. One would expect a stronger interaction of PLA-clay in PLA50, in which PLA chains were able to intercalate between clay platelets. However, this was not the case. We assume that the high amount of neighboring PLA chains provided an environment favorable for PLA-PLA interactions and consequently crystallization in PLA50. By contrast, the polymer chains in PLA20 were exhausted to "wet" clay particles, so that the possibility of interactions with other chains was minimized. After melting, 
reorganization of these clay-bonded chains occurred, maximizing interactions with the silicate and minimizing crystallization.

Due to this different behavior of PLA in PLA20 and PLA50, we decided to study the isothermal crystallization of PLA. Comparing DSC and FTIR results, it is clear that the response in terms of PLA-PLA and PLA-clay interactions was different for each case (Table 2, Table 3, and Figure 4). In PLA20, an increase in Si-O stretching vibrations related to a decrease in hydrogen bonding of clay with PLA, which must be the result of PLA crystallization and concomitant stronger PLA-PLA interactions. PLA50, where PLA was already weakly bonded to the clay, showed no difference in this vibration. By contrast, the carbonyl band showed a clear shift towards those of $\alpha^{\prime}$ crystals (main absorption band at $\sim 1759 \mathrm{~cm}^{-1}$ and a shoulder at $\sim 1749 \mathrm{~cm}^{-1} 15$ ). This was confirmed by the XRD spectrum of Figure 5, where the weak stereocomplex peak disappears and a somewhat broader peak, corresponding to the (200) reflection of $\alpha^{\prime}$ crystals, appears at $16,6^{\circ}$. This reflection also appeared in the spectrum of the annealed PLA20, although we detected only a very weak shoulder at $1750 \mathrm{~cm}^{-1}$ on the carbonyl stretching in Figure 4. The main cause for that is probably the low amount of PLA in the material and the low crystallinity of the specimens. The formation of $\alpha^{\prime}$ crystals in PLA50 and PLA100 was further confirmed by DSC.

The discussion so far clearly points to a change in the PLA20 system after annealing. While the as-prepared film showed strong interaction between polymer and clay, as well as a lack of melt crystallization, the annealed ones crystallized due to the reasons discussed previously. This crystallization was also accompanied by a decrease in the clay-polymer interaction, as given by the position of the Si-O stretching vibration. Interestingly, another effect was seen in this formulation (Figure 5): after annealing, we observed intercalation of PLA between clay layers. Not only intercalation occurred, but to the same extent as seen previously in PLA50 (that is, $d_{001}$ of about 3,0 nm). Intercalation requires extended mobility of chains over long distances, therefore it is not likely that intercalation occurred during isothermal cold crystallization. Instead, we propose the following explanation: PLA in PLA20 is located between clay platelets, but only as amorphous phase. In this case, chains are relatively mobile and may adopt a conformation so that they fit in the free volume of the interlayer, whereby polymer-clay interactions are favored. If sufficient time between $T_{g}$ and $T_{m}$ is allowed, chains will have enough mobility to pack together and crystallize. As a result, polymer-clay interactions will decrease and, due to the rigidity of crystallites, accommodation in the existing interlayer volume will be more difficult. 
The crystallites will then push the platelets apart, leading to an increase in $d_{001}$. This effect may be considered analogous to that observed with the stepwise increase in $d_{001}$ with the increase in the organic amount of the organoclay ${ }^{35}$ or with the chain length of the organic cation ${ }^{36}$. In fact, even with a fourfold increase in the polymer-to-clay ratio from PLA20 to PLA50, the $d_{001}$ of annealed PLA20 was the same as that for PLA50. Moreover, in the latter case no difference was found in $d_{001}$ before and after annealing. The amount of PLA was high enough so that it could not be accommodated in the original interlayer volume even as amorphous phase.

\section{Conclusion}

In this paper, we showed that biomimetic composites of PLA and organoclay may be prepared with high clay loading, yielding materials with improved barrier properties while still being transparent. A clay with a large basal spacing was more favorable to intercalation than the matching of the polarity between the polymer and the organic cation. We also investigated how the presence of the clay influences crystallization of PLA and, on the other hand, how the crystallization phenomenon influences the microstructure of the composite. We found that $50 \%$ of clay did not dramatically change the crystallization behaviour of PLA, while $80 \%$ of clay severely hindered crystallization of the polymer, specially melt crystallization. An interplay between the amount of PLA in the interlayer and the crystallinity affected the nanocomposite structure: simply by allowing amorphous PLA to crystallize in the interlayer volume of clay, an increase in the basal spacing was observed. This study allows a better understanding of the interaction between polymer and clay and may provide means for optimizing the structure and properties of highly loaded, biomimetic composites.

\section{Acknowledgements}

The authors thank Dr. Michael Plötze (ETHZ, Switzerland) for allowing the use of the XRD apparatus, and R. Hoffman and D. Sutter for help with the preparation of films.

\section{References}

[1] Endre, H.J. and Siebert-Raths, A. Technische Biopolymere. Hanser, München 2009.

[2] Thellen, C., Orroth, C., Froio, D., Ziegler, D., Lucciarini, J., Farrell, R., D’Souza, N. and Ratto, J. Polymer 2005, 46, 11716-11727. 
[3] Katiyar, V., Gerds, N., Koch, C., Risbo, J., Hansen, H. and Plackett, D. J. Appl. Polym. Sci. 2011, 122, $112-125$.

[4] Ray, S.S. and Bousmina, M. Prog. Mater. Sci. 2005, 50, 962-1079.

[5] Bharadwaj, R.K., Mehrabi, A.R., Hamilton, C., Trujillo, C., Murga, M., Fan, R., Chavira, A. and Thompson, A.K. Polymer 2002, 43, 3699-3705.

[6] Lu, C. and Mai, Y.W. In Physical properties and applications of polymer nanocomposites; Tjong, S.C.; Mai, Y.-W.; Eds.; Woodhead Publishing, Cambridge, UK, 2010; chap. 12, pp. 431-453.

[7] Stramm, C. Coating International 2005, 38, 263-265.

[8] Priolo, M.A., Gamboa, D., Holder, K.M. and Grunlan, J.C. Nano Lett. 2010, 10, 4970-4974.

[9] Priolo, M., Gamboa, D. and Grunlan, J. ACS Appl. Mater. Interfaces 2010, 2, 312-320.

[10] Walther, A., Bjurhager, I., Malho, J.M., Pere, J., Ruokolainen, J., Berglund, L.A. and Ikkala, O. Nano Lett. 2010, 10, 2742-2748.

[11] Pavlidou, S. and Papaspyrides, C.D. Prog. Polym. Sci. 2008, 33, 1119-1198.

[12] Krikorian, V. and Pochan, D.J. Macromolecules 2005, 38, 6520 - 6527.

[13] Pluta, M., Galeski, A., Alexandre, M., Paul, M.A. and Dubois, P. J. Appl. Polym. Sci. 2002, 86, $1497-$ 1506.

[14] Nam, J.Y., Ray, S.S. and Okamoto, M. Macromolecules 2003, 36, 7126-7131.

[15] Pan, P., Zhu, B., Kai, W., Dong, T. and Inoue, Y. Macromolecules 2008, 41, 4296-4304.

[16] Garlotta, D. J. Polym. Environ. 2001, 9, 63-84.

[17] Yao, H.B., Tan, Z.H., Fang, H.Y. and Yu, S.H. Angew. Chem., Int. Ed. 2010, 49, 10127-10131.

[18] Wool, R.P. In Bio-based polymers and composites; Wool, R.P.; Sun, R.P.; Eds.; Elsevier, Amsterdam, 2005; chap. 15, pp. 523-550.

[19] Karst, D. and Yang, Y. J. Appl. Polym. Sci. 2005, 96, 416-422.

[20] Ojijo, V., Cele, H. and Ray, S.S. Macromol. Mater. Eng. 2011, 296, 865-877.

[21] Cervantes-Uc, J.M., Cauich-Rodrí̂A-guez, J.V., Vázquez-Torres, H., Garfias-Mesías, L.F. and Paul, D.R. Thermochim. Acta 2007, 457, 92-102.

[22] Walther, A., Bjurhager, I., Malho, J.M., Ruokolainen, J., Berglund, L. and Ikkala, O. Angew. Chem., Int. Ed. 2010, 49, $6448-6453$.

[23] Pluta, M., Paul, M.A., Alexandre, M. and Dubois, P. J. Polym. Sci., Part B: Polym. Phys. 2006, 44, 299-311.

[24] Wu, D., Wu, L., Wu, L., Xu, B., Zhang, Y. and Zhang, M. J. Nanosci. Nanotechnol. 2008, 8, 16581668.

[25] Wu, D., Wu, L., Wu, L., Xu, B., Zhang, Y. and Zhang, M. J. Polym. Sci., Part B: Polym. Phys. 2007, $45,1100-1113$.

[26] Li, S. and Vert, M. Polym. Int. 1994, 33, $37-41$.

[27] Li, S. and McCarthy, S. Biomaterials 1999, 20, 35-44.

[28] Martino, V., Ruseckaite, R. and Jiménez, A. Polym. Int. 2009, 58, 437-444.

[29] Meaurio, E., Zuza, E., López-Rodríguez, N. and Sarasua, J.R. J. Phys. Chem. B 2006, 110, 5790-5800.

[30] Meaurio, E., López-Rodríguez, N. and Sarasua, J.R. Macromolecules 2006, 39, 9291-9301.

[31] Sarasua, J.R., López Rodríguez, N., López Arraiza, A. and Meaurio, E. Macromolecules 2005, 38, 8362-8371.

[32] Kister, G., Cassanas, G. and Vert, M. Polymer 1998, 39, 267-273.

[33] Tsuji, H. Macromol. Biosci. 2005, 5, 569-597.

[34] Yan, L., Roth, C.B. and Low, P.F. Langmuir 1996, 12, 4421-4429.

[35] Boesel, L.F. and Pessan, L.A. Mater. Sci. Forum 2002, 403, 89-94.

[36] Heinz, H., Vaia, R.A., Krishnamoorti, R. and Farmer, B.L. Chem. Mater. 2007, 19, 59-68.

\section{Supplementary Info}

Videos showing the behavior of pure PLA and PLA/43B composite when in contact with a flame. 
1 SEM images of the cross section of (a) a pure PLA film, and (b) a composite of $20 \%$ PLA and $80 \%$ clay. c) Optical images of composite films, showing that composites with high clay loading are still transparent: the numbers (under the films) indicate the amount of clay of each film. 16

2 XRD spectra of clays I34 and 43B (solid lines) and composites thereof with PLA (dotted lines). The peak at about $9,5^{\circ}$ corresponds to talc, used as internal reference. $d_{001}$ is indicated above the (001) reflections for each material. 17

3 TGA curves of PLA, organoclays and composites thereof. .18

4 Detailed view of FTIR spectra of formulations before (solid lines) and after (dotted lines) annealing at $130^{\circ} \mathrm{C}$. a) $\mathrm{C}=\mathrm{O}$ stretching vibration; b) Si-O stretching vibration.

5 XRD spectra of formulations before (solid lines) and after (dotted lines) annealing at $130{ }^{\circ} \mathrm{C}$. Peaks at $9,5^{\circ}, 19^{\circ}$, and $28,7^{\circ}$ are from talc, used as a reference. Other main peaks: PLA stereocomplex $\left(21,5^{\circ}\right.$ and $\left.24^{\circ}\right), \alpha^{\prime}$-crystal of PLA $\left(16,6^{\circ}\right.$ and $\left.18,9^{\circ} *\right),(001)$ reflections of organoclay $\left(4,2^{\circ}\right)$ and composites $\left(\sim 2,8^{\circ}\right)$. * This peak may only be observed for PLA $100 \%$ after annealing; for other samples it is masked by the peak of talc at $19^{\circ}$. 20

6 DSC curves of PLA/43B 50/50 composites before (first and second heating) and after (only first heating) annealing at $130{ }^{\circ} \mathrm{C}$. Inset: melting peak of pure PLA during first (solid line) and second (dotted line) heating runs.

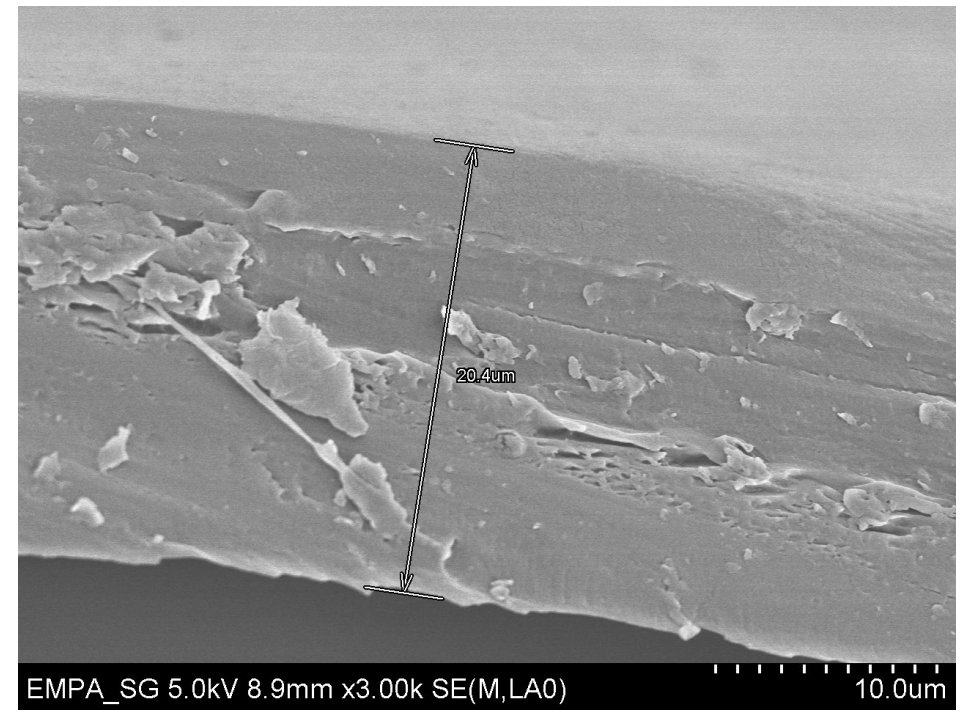



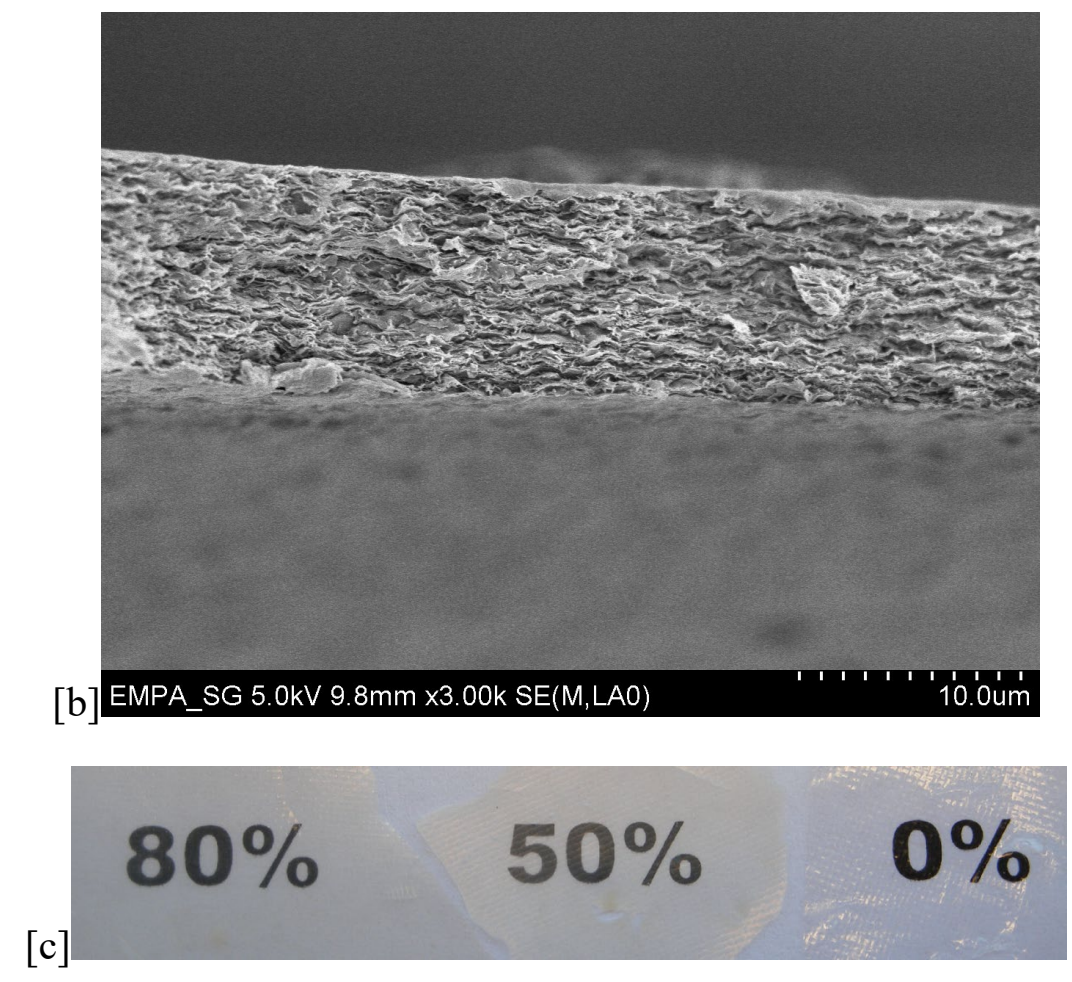

Figure 1: SEM images of the cross section of (a) a pure PLA film, and (b) a composite of $20 \%$ PLA and $80 \%$ clay. c) Optical images of composite films, showing that composites with high clay loading are still transparent: the numbers (under the films) indicate the amount of clay of each film. 


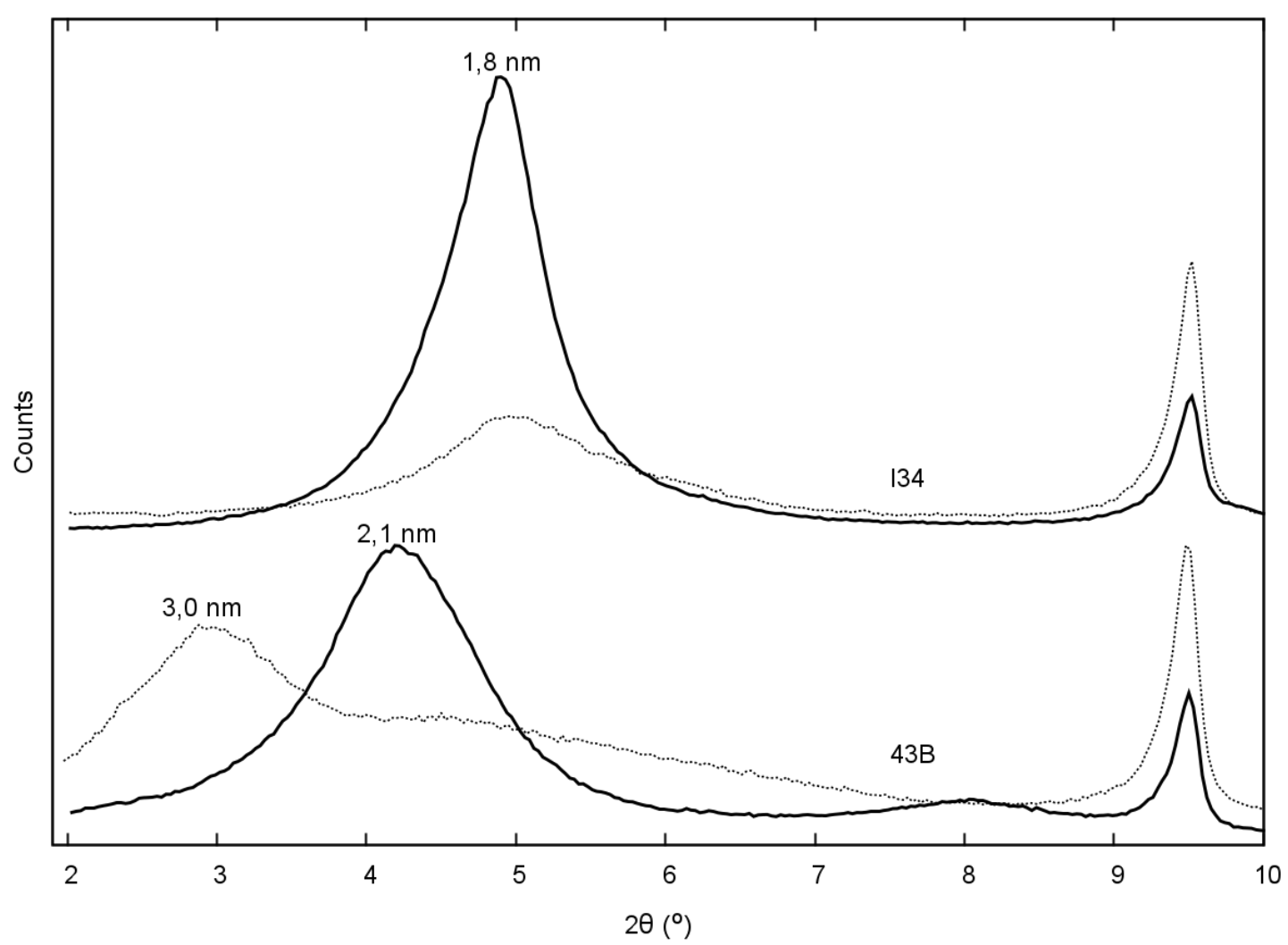

Figure 2: XRD spectra of clays I34 and 43B (solid lines) and composites thereof with PLA (dotted lines). The peak at about $9,5^{\circ}$ corresponds to talc, used as internal reference. $d_{001}$ is indicated above the (001) reflections for each material. 


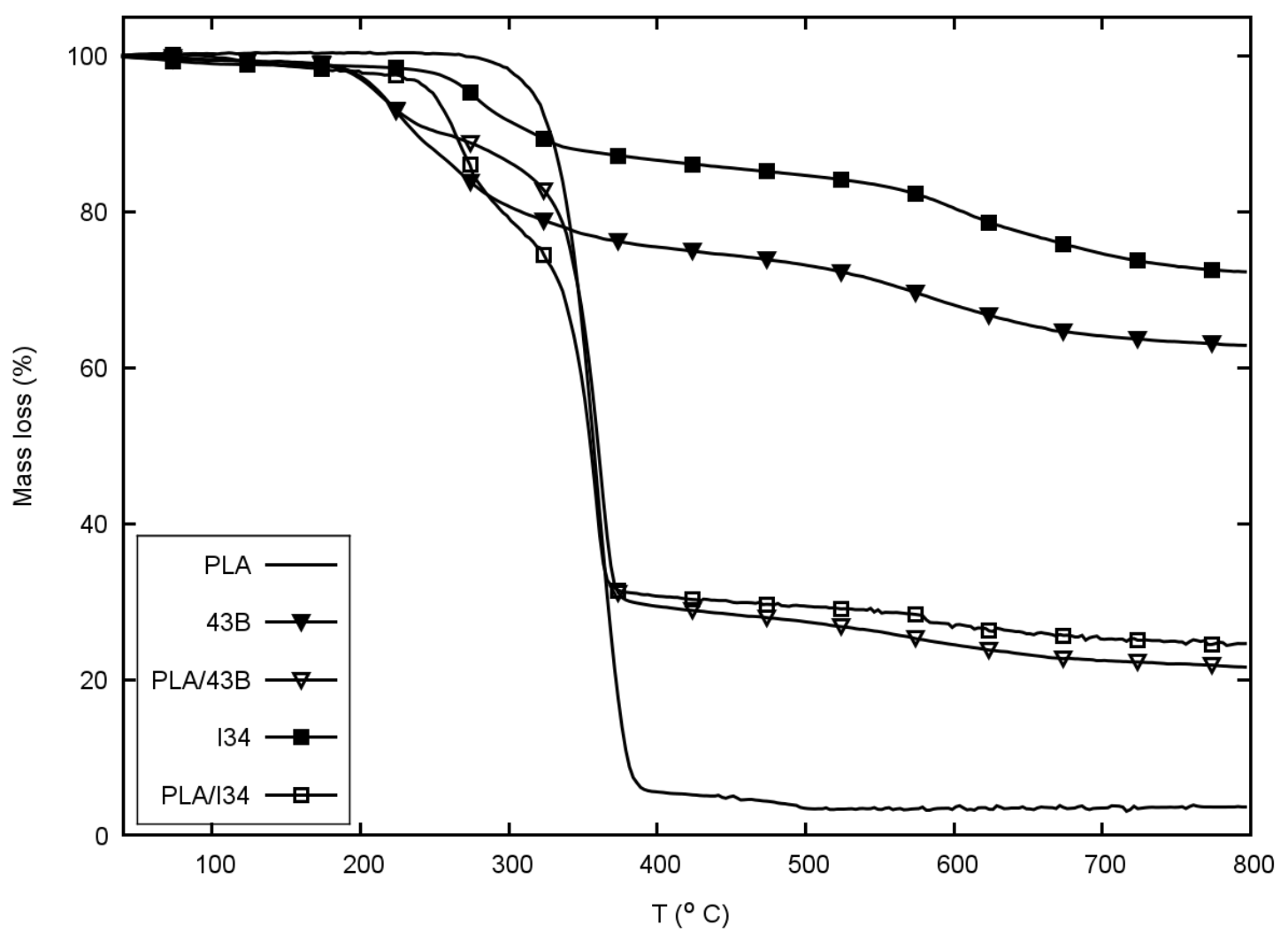

Figure 3: TGA curves of PLA, organoclays and composites thereof. 


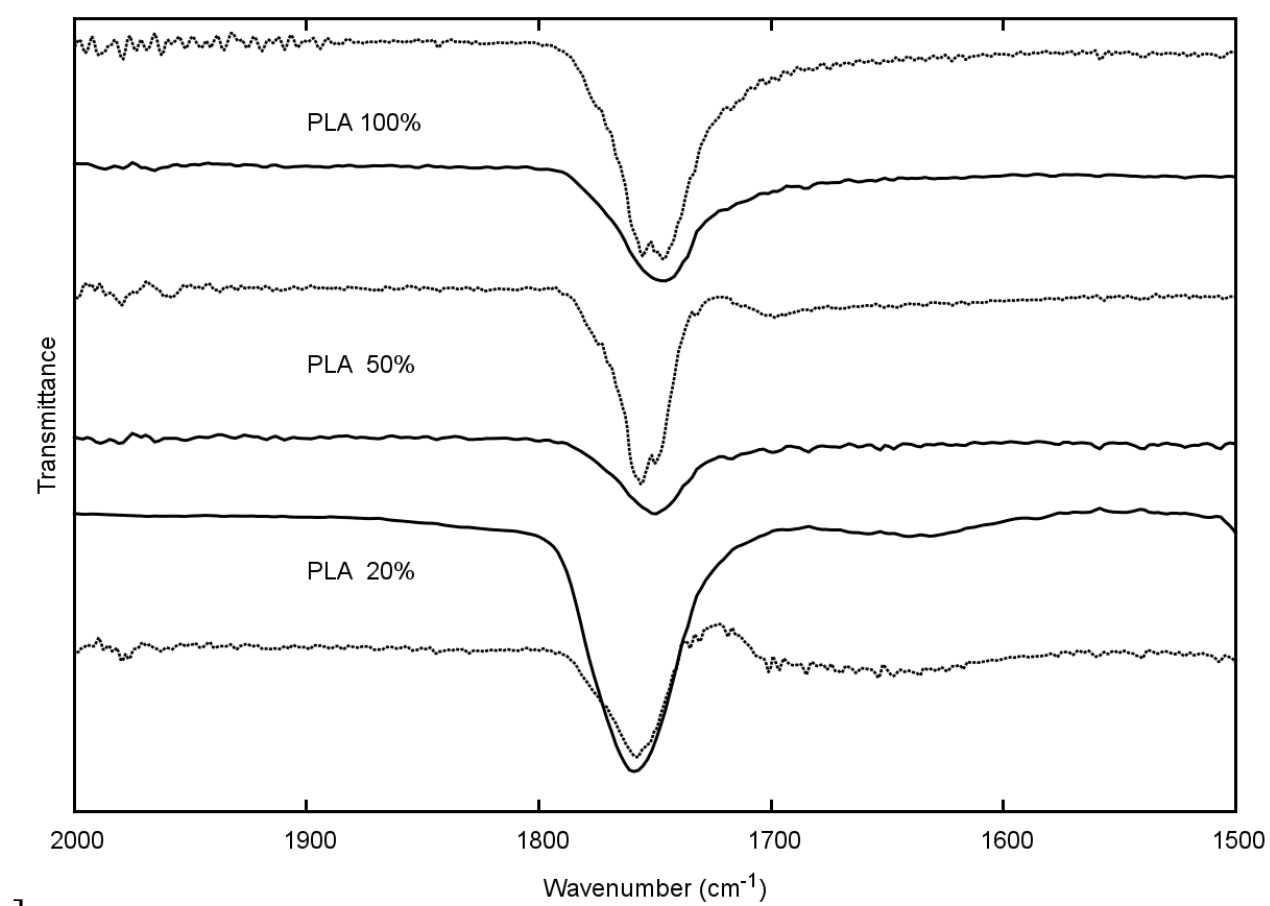

[a]

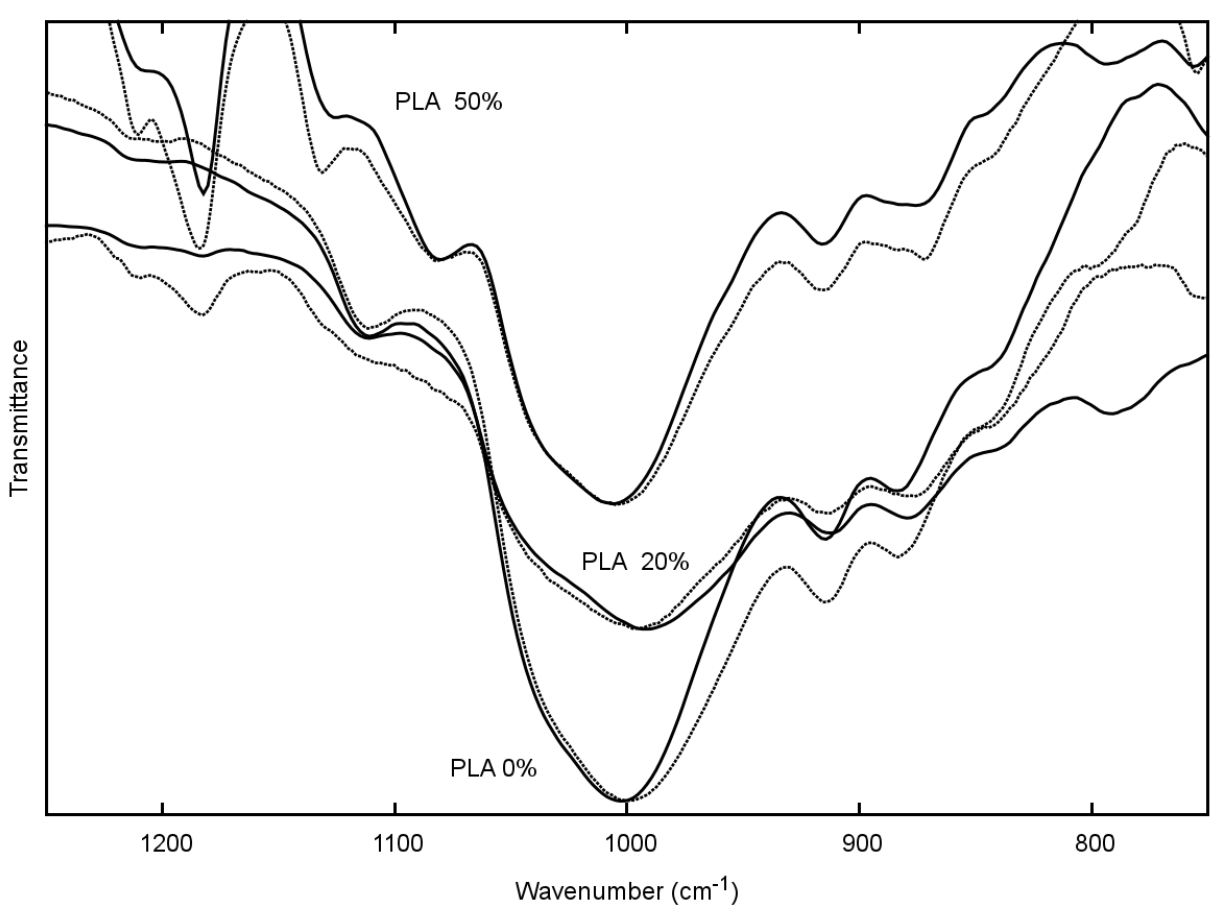

[b]

Figure 4: Detailed view of FTIR spectra of formulations before (solid lines) and after (dotted lines) annealing at $130{ }^{\circ} \mathrm{C}$. a) $\mathrm{C}=\mathrm{O}$ stretching vibration; b) Si-O stretching vibration. 


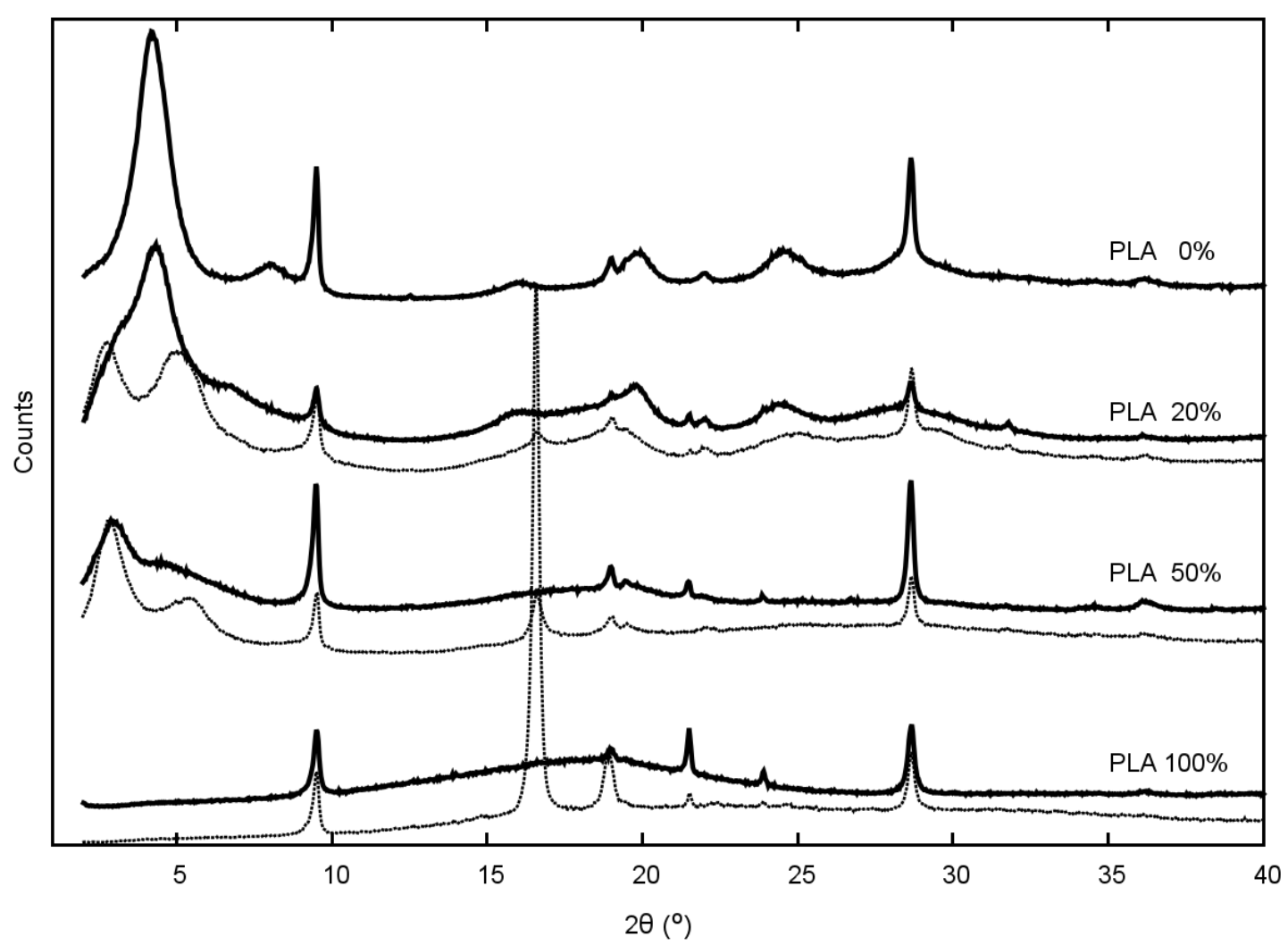

Figure 5: XRD spectra of formulations before (solid lines) and after (dotted lines) annealing at $130^{\circ} \mathrm{C}$. Peaks at $9,5^{\circ}, 19^{\circ}$, and $28,7^{\circ}$ are from talc, used as a reference. Other main peaks: PLA stereocomplex $\left(21,5^{\circ}\right.$ and $\left.24^{\circ}\right), \alpha^{\prime}$-crystal of PLA $\left(16,6^{\circ}\right.$ and $\left.18,9^{\circ} *\right),(001)$ reflections of organoclay $\left(4,2^{\circ}\right)$ and composites $\left(\sim 2,8^{\circ}\right)$. ${ }^{*}$ This peak may only be observed for PLA $100 \%$ after annealing; for other samples it is masked by the peak of talc at $19^{\circ}$. 


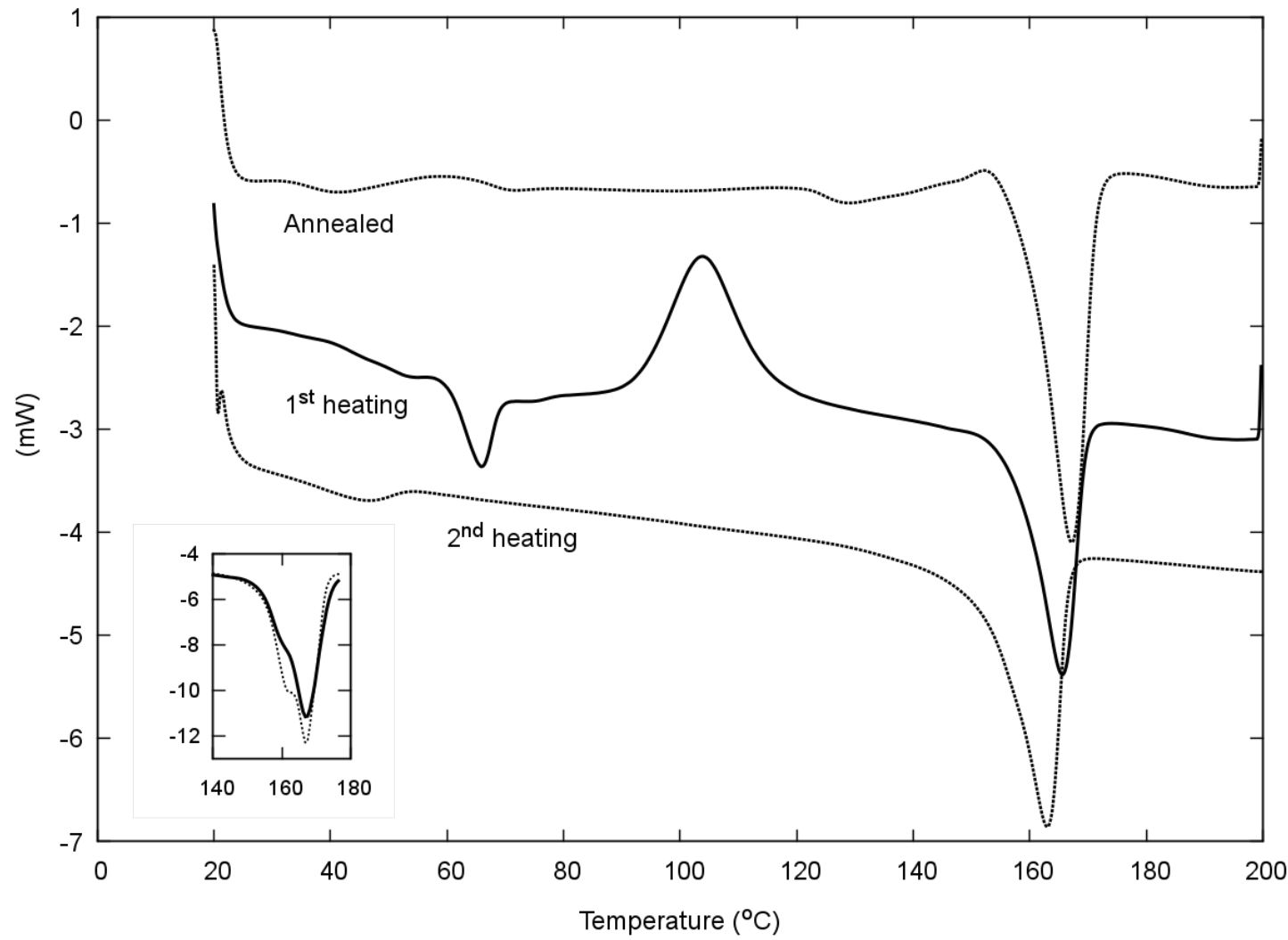

Figure 6: DSC curves of PLA/43B 50/50 composites before (first and second heating) and after (only first heating) annealing at $130{ }^{\circ} \mathrm{C}$. Inset: melting peak of pure PLA during first (solid line) and second (dotted line) heating runs. 
1 Water vapor transmission rate of PLA and composites thereof...............22

2 Changes in FTIR spectrum as function of annealing and clay content.....22

3 Thermal properties of composites before and after annealing, obtained during the first heating, except where indicated otherwise. ................................................22

Table 1: Water vapor transmission rate of PLA and composites thereof.

\begin{tabular}{c|c}
\hline Material & WVTR in [Fehler!] \\
\hline PLA & $15 \pm 7,0$ \\
PLA/43B 20/80 & $1,4 \pm 1,2$ \\
PLA/43B 50/50 & $6,0 \pm 3,6$ \\
PLA/I34 50/50 & $5,5 \pm 2,9$ \\
Coating (PLA/I34 50/50 over PLA) & $6,3 \pm 2,1$ \\
\hline
\end{tabular}

Table 2: Changes in FTIR spectrum as function of annealing and clay content.

\begin{tabular}{c|c|cc|cc}
\hline Formulation & PLA (\%) & \multicolumn{2}{c|}{$\begin{array}{c}\mathrm{C}=\mathrm{O} \text { stretching (PLA), } \\
\text { in } \mathrm{cm}^{-1}\end{array}$} & \multicolumn{2}{c}{$\begin{array}{c}\text { Si-O stretching } \\
\text { (clay), } \\
\text { in cm }\end{array}$} \\
\hline PLA100 & & Before & After & Before & After \\
\hline PLA50 & 100 & 1746 & $1755 / 1746$ & - & - \\
PLA20 & 50 & 1750 & $1756 / 1749$ & 1006 & 1004 \\
PLA0 or 43B & 20 & 1758 & $1758 / 1750$ & 992 & 997 \\
& 0 & - & - & 1002 & 999 \\
\hline
\end{tabular}

Table 3: Thermal properties of composites before and after annealing, obtained during the first heating, except where indicated otherwise.

\begin{tabular}{l|l|l}
\hline & Before annealing & After annealing \\
\hline
\end{tabular}




\begin{tabular}{c|ccc|ccc} 
PLA (\%) & $T_{g}\left({ }^{\circ} \mathrm{C}\right)$ & $T_{m}\left({ }^{\circ} \mathrm{C}\right)$ & $\chi_{c}(\%)$ & $T_{g}\left({ }^{\circ} \mathrm{C}\right)$ & $T_{m}\left({ }^{\circ} \mathrm{C}\right)$ & $\chi_{c}(\%)$ \\
\hline 100 & 53 & 167 & 44 & 65 & 168 & 45 \\
50 & 63 & 166 & 45 & 68 & 167 & 36 \\
20 & 60 & 164 & - & 65 & 164 & 16 \\
\hline
\end{tabular}

* calculated from the second heating curve. 